\title{
Reconocimiento de emociones a partir de descripciones verbales
}

\author{
ALFONSO JIMÉNEZ FERNÁNDEZ, \\ M. J JOSÉ MALLO CARRERA \\ Departamento de Psicologia Básica, Social y Metodológica. \\ Universidad Autónoma de Madrid.
}

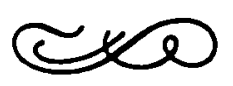

\section{Resumen}

El presente estudio supone una aplicación tentativa de la técnica del autoinforme a la codificación y decodificación de las manifestaciones corporales y expresivas asociadas al comportamiento emocional. El interés en el uso de dicha técnica radica, no tanto en la posibilidad de determinar los cambios reales asociados a la emoción, como de conocer el modo en que los sujetos recuerdan y categorizan su experiencia emocional.

\section{Abstract}

This study explores the role of questionnaries in the research dealing with the recognition of emotional expression. The goal of our research is to stablish the patterns of categorization of physiological, verbal and expressive manifestations of emotional experience.

Dirección de los autores: Departamento de Psicologia Básica Social y Metodologia. Fac. Psicología Universidad Autónoma de Madrid. 28049 Madrid.

Esta investigación ha sido parcialmente subvencionada por el proyecto PB85-0226 de la Comisión Asesora para la investigación Científica y Técnica. 


\section{INTRODUCCION}

En el estudio de la emoción siempre se han considerado las alteraciones corporales como componentes importantes de las experiencias emocionales, y esto se refleja así ya en los trabajos clásicos sobre las emociones (por ejemplo, James, 1884; Wundt, 1905; Cannon, 1915). El papel que tiene el componente fisiológico sobre la experiencia emocional ha sido uno de los temas más discutidos de los investigadores de la emoción (Fernández Dols y Ortega, 1984), especialmente, a raiz de las discusiones sobre la especifidad de los distintos estados emocionales y sobre las interrelaciones entre los cambios fisiológicos y los aspectos más cognitivos de la emoción, discusiones que en sus orígenes ya enfrentaron a James y Cannon y que se han venido manteniendo desde aquellos momentos, en diferentes polémicas (por ejemplo, Zajonc, 1980 vs., Lazarus, 1982; Valle Inclán, 1983, 1985 vs., Iglesias y col., 1984). Algunas de estas polémicas, como el caso de la universalidad o la posible transmisión innata de la emoción, han sido favorecidas por otra línea paralela de investigación y que tiene su origen en el trabajo de Darwin (1872), se trata del estudio de la expresión de la emoción, habiéndose concentrado las investigaciones posteriores en el estudio de la expresión facial (Tomkins, 1962; Izard, 1971; Ekman, 1972), y en algunos casos en otros canales expresivos como el vocal (Jiménez, en prensa). Además de estas aproximaciones generales, otros investigadores que han incidido en otros componentes emocionales, tal es el caso de sociológos, como Kemper (1978) o Torregrosa (1984) que hacen especial hincapié en los aspectos sociales y culturales que desencadenan las experiencias emocionales. Igualmente, otras teorías psicológicas (por ejemplo, el conductismo o el psicoanálisis) también se han acercado al tema desde presupuetos distintos.

Paralelamente a la discusión teórica, se han utilizado diversas técnicas de investigación en cada una de las grandes aproximaciones al estudio de la emoción, así mientras las investigación fisiológica se ha centrado en los registros fisiológicos de señales en situaciones de laboratorio, la línea expresiva ha trabajado en taxonomías de unidades expresivas a partir de técnicas de observación empleando jueces, más o menos expertos.

Como se ve existe una gran cantidad de formas de entender e investigar las emociones, esto ha llevado a algunos autores como Plutchik y Kellerman (1980) a considerar necesaria una teoría integradora de todas las aproximaciones existentes, aceptando los datos y resultados más sobresalientes de cada una. Así surge en el espectro teórico de la emoción la teoría de los procesos componentes de Scherer $(1982,1984)$. Para Scherer existirían cinco componentes dinámicos: un componente evaluativo de la situación ambiental, otro motivacional, otro expresivo (verbal y no verbal), el componente fisiológico y un componente subjetivo del estado.

Difícilmente se podrán evaluar todos los componentes si no empleamos alguna técnica de autoinforme con sus conocidas ventajas e inconvenientes (Scherer, 1968b). Así surgió el proyecto internacional ESI (Emotion in Social Interaction), que agrupó a diversos investigadores de ocho países europeos y que aplicó un cuestionario que interrogaba sobre cuatro estados emocionales (alegría, tristeza, miedo y cólera) a una muestra de 779 estudiantes universitarios cuyas características muestrales son descritas por 
Aebischer y Wallbott (1986). Dentro del cuestionario se pedía a los sujetos que evocaran una situación en la que hubiesen experimentado cada uno de los estados emocionales descritos. Posteriormente, se preguntaba sobre las expresiones y cambios corporales experimentados, utilizando un formato abierto cuya categorización se realizó mediante el código descrito por Ellgring y Bänninger-Huber (1986). A partir de estos resultados se determinaron las expresiones verbales (Cosnier, Fernández Dols y Jiménez, 1986), no verbales (Wallbott, Ricci-Bitti y Bänninger-Huber, 1986) y los síntomas corporales (Rimé y Giovannini, 1986) que con mayor frecuencia indicaban los sujetos.

\section{METODO Y RESULTADOS DEL PRIMER ESTUDIO}

El trabajo anterior, recogido en una compilación de Scherer, Wallbott y Summerfield (1986), dio pie al diseño de un nuevo proyecto internacional de investigación: ISEAR (International Survey on Emotion: Antecedents and Reactions), en el que nuestro equipo también está integrado. Dicho proyecto mejoró el cuestionario con la experiencia de los resultados del proyecto ESI y se está aplicando a muestras de universitarios de varios países, habiéndose aumentado el número de emociones a investigar con la

\section{TABLA I}

Categorias de respuesta of recidas a los sujetos en el cuestionario ISEAR (el nombre abreviado de cada manifestación servirá para la lectura de las tablas posteriores)

\begin{tabular}{llr}
\hline Cambios corporales & Nudo en la garganta & $1 \mathrm{~B}$ \\
& Alteración ritmo respiratorio & $2 \mathrm{~B}$ \\
& Molestias de estómago & $3 \mathrm{~B}$ \\
& Sensación de frío, tiritar & $4 \mathrm{~B}$ \\
& Sensación cálida, agradable & $5 \mathrm{~B}$ \\
& Sensación de calor, mejillas sonrojadas & $6 \mathrm{~B}$ \\
& Aceleración ritmo cardíaco & $7 \mathrm{~B}$ \\
& Tensión muscular, temblor & $8 \mathrm{~B}$ \\
& Relajación muscular, sosiego & $9 \mathrm{~B}$ \\
& Sudar, manos sudorosas & $10 \mathrm{~B}$ \\
& Otros síntomas & $11 \mathrm{~B}$ \\
Expresiones no verbales & Reír, sonreír & $1 \mathrm{E}$ \\
& Llorar, sollozar & $2 \mathrm{E}$ \\
& Cambios de expresión facial & $3 \mathrm{E}$ \\
& Chillar, gritar & $4 \mathrm{E}$ \\
& Cambios en la voz & $5 \mathrm{E}$ \\
& Cambios gestuales & $6 \mathrm{E}$ \\
& Cambios corporales bruscos & $7 \mathrm{E}$ \\
& Avanzar hacia personas o cosas & $8 \mathrm{E}$ \\
& Alejarse de personas o cosas & $9 \mathrm{E}$ \\
& Agredir a personas o cosas & $10 \mathrm{E}$ \\
& Otras reacciones expresivas & $11 \mathrm{E}$ \\
& Silencio & $1 \mathrm{~V}$ \\
& Expresión corta & $2 \mathrm{~V}$ \\
& Una o dos frases & $3 \mathrm{~V}$ \\
& Expresión larga & $4 \mathrm{~V}$ \\
& Cambio en la melodia del habla & $5 \mathrm{~V}$ \\
& Perturbaciones en el habla & $6 \mathrm{~V}$ \\
& Cambios en el ritmo del habla & $7 \mathrm{~V}$ \\
& Otras reacciones verbales & $8 \mathrm{~V}$ \\
\hline & & \\
& &
\end{tabular}


incorporación de asco, vergüenza y culpa a las cuatro del proyecto anterior. Respecto a las preguntas referidas a expresiones verbales, no verbales y cambios corporales, en el cuestionario ISEAR se presentan diversas categorías nominales no excluyentes que los sujetos pueden elegir para identificar cada uno de sus estados emocionales. Dichas categorías pueden verse en la tabla I.

En nuestro país se aplicó a una muestra de 83 estudiantes universitarios de las distintas facultades de la Universidad Autónoma de Madrid.

TABLA II

Resultados de cambios corporales y expresiones (verbales $y$ no verbales) de la muestra española tras la aplicación del cuestionario ISEAR (las proporciones marcadas en negrita son los cambios señalados con mayor frecuencia en cada emoción)

\begin{tabular}{|c|c|c|c|c|c|c|c|c|c|c|c|}
\hline \multirow[b]{2}{*}{ Emoción } & \multicolumn{11}{|c|}{ CAMBIOS CORPORALES } \\
\hline & 1B & 2B & 3B & 4B & $5 B$ & $6 B$ & $7 \mathrm{~B}$ & 8B & 9B & $10 \mathrm{~B}$ & $11 B$ \\
\hline Alegría & .13 & .26 & .01 & .05 & .47 & .13 & .41 & .10 & .24 & .11 & .14 \\
\hline Miedo & .36 & .42 & .14 & .22 & .00 & .06 & .60 & .52 & .00 & .30 & .11 \\
\hline Cólera & .23 & .42 & .11 & .04 & .01 & .26 & .47 & .45 & .00 & .19 & .18 \\
\hline Tristeza & .49 & .11 & .20 & .19 & .01 & .05 & .08 & .17 & .08 & .08 & .29 \\
\hline Asco & .13 & .13 & .46 & .06 & .00 & .05 & .12 & .23 & .01 & .08 & .34 \\
\hline Vergüenza & .28 & .31 & .06 & .02 & .04 & .60 & .41 & .20 & .00 & .24 & .07 \\
\hline Culpa & .41 & .16 & .17 & .06 & .00 & .12 & .18 & .22 & .01 & .24 & .20 \\
\hline
\end{tabular}

TABLA II (cont.)

\begin{tabular}{lccccccccccc}
\hline & \multicolumn{10}{c}{ EXPRESIONES NO VERBALES } \\
\cline { 2 - 11 } \multicolumn{1}{c}{ Emoción } & 1E & 2E & 3E & 4E & 5E & 6E & 7E & 8E & 9E & 10E & 11E \\
\hline Alegría & $\mathbf{8 8}$ & .08 & .10 & .19 & .07 & .25 & .06 & .29 & .01 & .00 & .06 \\
Miedo & .06 & .12 & .29 & .13 & .11 & .31 & .13 & .14 & .17 & .00 & .18 \\
Cólera & .02 & .12 & .30 &. $\mathbf{4 3}$ & .19 & .32 & .22 & .17 & .11 & .16 & .16 \\
Tristeza & .01 & .47 & .25 & .02 & .08 & .13 & .08 & .05 & .13 & .00 & .23 \\
Asco & .05 & .00 & $\mathbf{. 4 8}$ & .11 & .04 & .30 & .12 & .05 & .19 & .04 & .14 \\
Vergüenza & .24 & .02 & .29 & .02 & .20 & .34 & .07 & .03 & .14 & .04 & .13 \\
Culpa & .04 & .20 & .30 & .11 & .17 & .19 & .10 & .05 & .23 & .00 & .13 \\
\hline
\end{tabular}

TABLA II (cont.)

\begin{tabular}{lcccccccc}
\hline & \multicolumn{8}{c}{ EXPRESIONES VERBALES } \\
\cline { 2 - 8 } \multicolumn{1}{c}{ Emoción } & $\mathbf{1 V}$ & $\mathbf{2 V}$ & $\mathbf{3 V}$ & $\mathbf{4 V}$ & $\mathbf{5 V}$ & $\mathbf{6 V}$ & $\mathbf{7 V}$ & $\mathbf{8 V}$ \\
\hline Alegría & .07 & .20 & .11 & $\mathbf{. 3 0}$ & .29 & .08 & .20 & .17 \\
Miedo & $\mathbf{4 8}$ & .25 & .08 & .10 & .08 & .12 & .12 & .04 \\
Cólera & .15 & .15 & .13 & .35 & .16 & .14 & .29 & .16 \\
Tristeza & $\mathbf{. 8 2}$ & .19 & .04 & .07 & .13 & .11 & .11 & .01 \\
Asco & $\mathbf{3 4}$ &. $\mathbf{2 8}$ & .13 & .10 & .06 & .05 & .05 & .12 \\
Vergüenza & $\mathbf{. 4 3}$ & $\mathbf{. 2 7}$ & $\mathbf{. 1 7}$ & .03 & .16 & .14 & .02 & .04 \\
Culpa & $\mathbf{. 5 9}$ &. $\mathbf{2 0}$ & .10 & .08 & .06 & .10 & .10 & .05 \\
\hline
\end{tabular}


Los resultados resumidos en la tabla II, muestran claras diferencias para cada emoción; sin embargo, algunos cambios corporales o expresiones verbales o no verbales son comunes para varias emociones. Los cambios más importantes que los sujetos informan respecto a situaciones en las que experimentaron alegría fueron, en orden de mayor a menor proporción de elección: reír (.88), sensación cálida (.47), aceleración del ritmo cardiaco (.41) y hablar (.30). En el caso de miedo: aceleración del ritmo cardiaco (.60), tensión muscular (.52), silencio (.48) y cambios en la respiración (.42). Para cólera: aceleración del ritmo cardiaco (.47), tensión muscular (.52), chillar (.43) y cambios en la respiración (.42). Para tristeza: silencio (.82), llorar (.47), sentir un nudo en la garganta (.49) y cambios en la expresión facial (.25). Para asco: cambios en la expresión facial (.48), molestias en el estómago (.46), silencio (.34), y cambios gestuales (.30). En el caso de vergüenza: mejillas sonrojadas (.60), silencio (.43), aceleración ritmo cardiaco (.41) y cambios gestuales (.34). Por último asociados a culpa se indicaron: silencio (.59), nudo en la garganta (.41), cambios en la expresión facial (.30) y manos sudorosas (.24).

Como se ve, existen cambios que son referidos por un alto porcentaje de sujetos en varias emociones: aceleración del ritmo cardiaco (alegría, miedo, cólera y vergüenza), silencio (miedo, tristeza, asco, vergüenza y culpa), tensión muscular (miedo y cólera), cambios en la respiración (miedo y cólera), entre otros.

FIGURA I

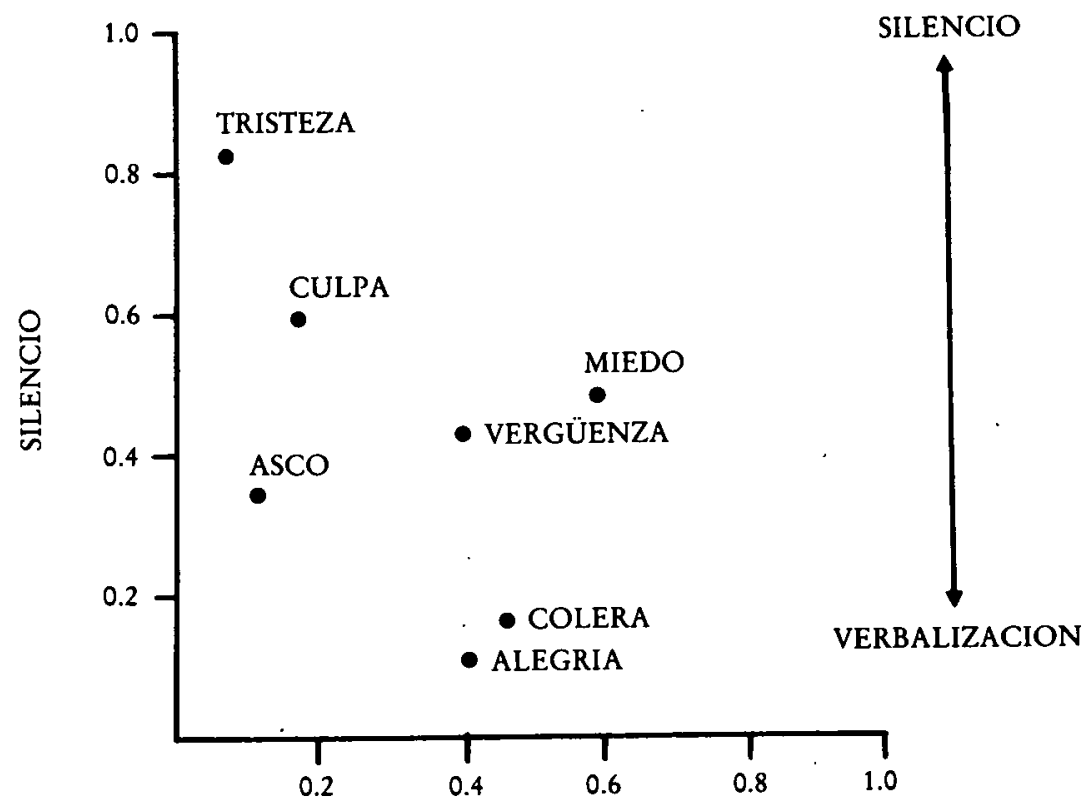

ACELERACION RITMO CARDIACO

Media de los cambios referidos en cada emoción para cada tipo de manifestaciones: corporales $y$ expresivos (no verbales $y$ verbales) 
Calculamos la media de cambios, de cada uno de los tres tipos indicados por los sujetos, observándose (ver figura 1) grandes diferencias entre emociones. Así, miedo, cólera y vergüenza son las emociones que incluyen en su caracterización un mayor número de cambios corporales, en este sentido podrán ser consideradas emociones que se categorizan y recuerdan en términos preferentemente fisiológicos o internos. Sin embargo, en el caso, de cólera, también pueden observarse altas puntuaciones en el número de cambios expresivos, por lo cual se calculó un índice de externalización de la emoción dividiendo a la media de cambios expresivos no verbales la media de cambios corporales, así se representan en la figura 2 las puntuaciones de dicho índice para cada una de las siete emociones investigadas. Se observa como alegría es la emoción más externalizada mientras que miedo es la más internalizada.

FIGURA II

\section{INDICE DE EXTERIORIZACION/INTERIORIZACION}

$\mathrm{i}=$ media (categorías externas)/media (categorías internas)

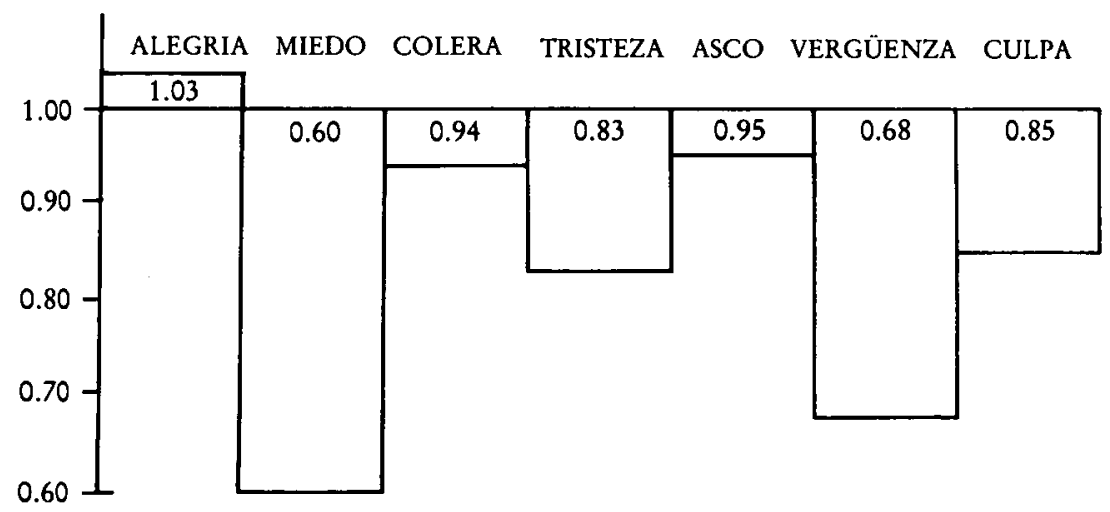

Representación de los indices de externalización/internalización para cada emoción ( $i=$ media de categorias no verbales/media de cambios corporales)

Por otra parte, a partir de las proporciones obtenidas en dos cambios repetidamente descritos para varias emociones, ritmo cardiaco y silencio, un cambio corporal y la ausencia de manifestación verbal, hemos construido una figura bidimensional en la que se sitúan las siete emociones investigadas. Como se puede observar en la figura 3 , en cuanto a la aceleración del ritmo cardiaco las emociones de miedo, cólera, alegría, y vergüenza muestran altos porcentajes mientras que en tristeza, asco y culpa son menores; silencio tiene bajos porcentajes únicamente en alegría y cólera, de modo que si consideramos dichos cambios como representantes de las dimensiones activación y verbalización respectivamente se configurarían tres grupos de emociones en función de estos resultados: no silenciosas y con activación, silenciosas y activas, y silenciosas y con baja activación. 
Figura III
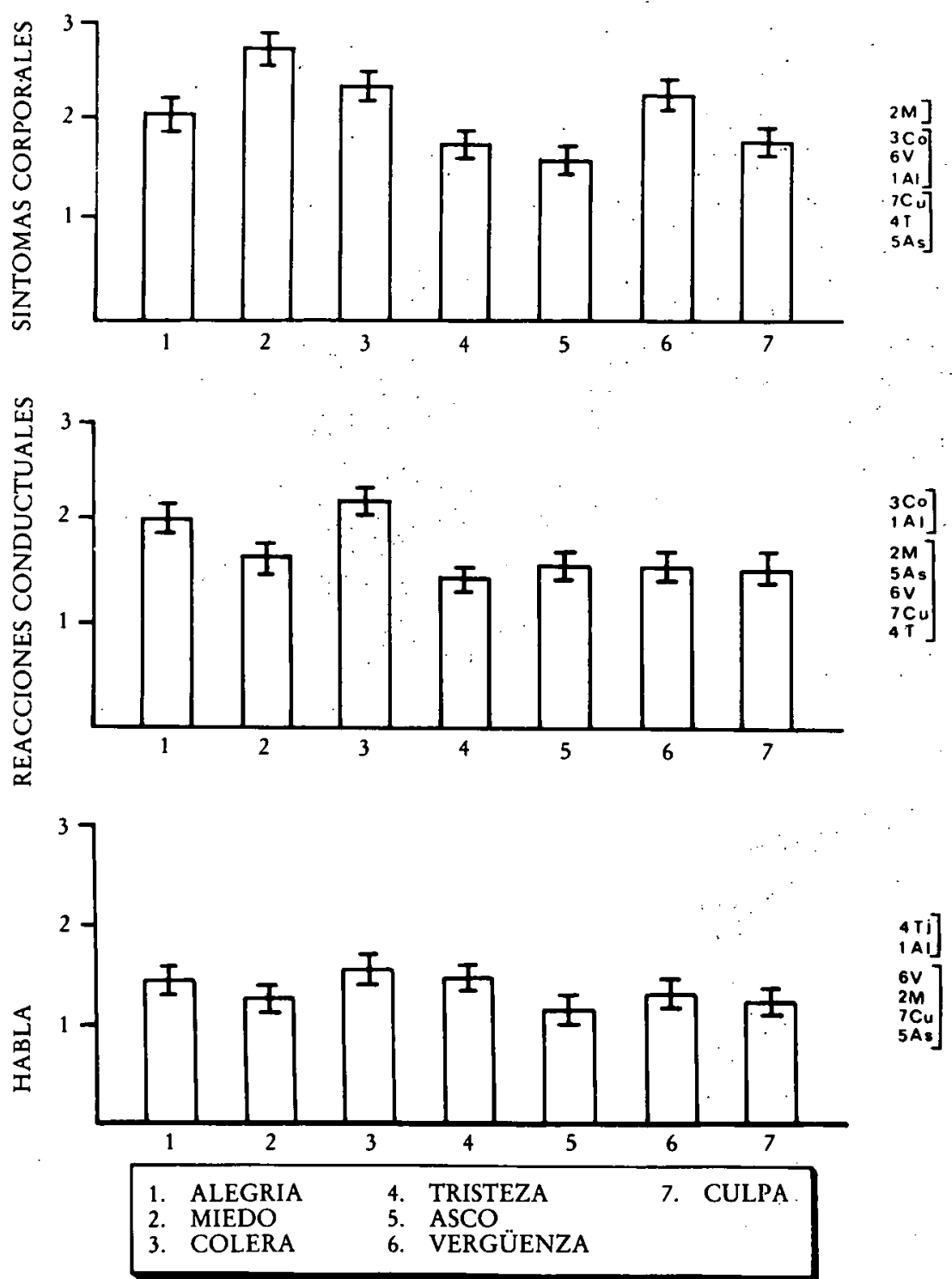

Representación bidimensional de las siete emociones investigadas en función de la proporción de las variables aceleración del ritmo cardiaco (abscisa) y silencio (ordenada)

\section{METODO Y RESULTADOS DEL SEGUNDO ESTUDIO}

A la vista de estos resultados nos planteamos la posibilidad de aplicar la metodología de reconocimiento, tradicional en la investigación expresiva, en este campo; de modo que presentamos a una muestra de 54 estudiantes universitarios de nuestra Facultad, las descripciones verbales de los cambios más informados en el estudio primero, bien independientes o en 
combinaciones de dos, tres y cuatro cambios, a los que los sujetos deberían asignar una de las siete emociones investigadas.

Los resultados a partir de combinaciones de cuatro manifestaciones muestran (ver tabla III) que existe un alto porcentaje de reconocimiento emocional. Unicamente en el caso de culpa el reconocimiento parece resultar problemático (.41), identificándose como miedo en un alto porcentaje de ocasiones.

\section{TABLA III}

Matriz completa de datos con las proporciones de elección de cada emoción para las combinaciones de cuatro manifestaciones (señaländose en negrita los valores que representan las opciones correctas de respuesta)

\begin{tabular}{lrrrrrrrrr}
\hline \multirow{2}{*}{$\begin{array}{c}\text { Categoría } \\
\text { elegida }\end{array}$} & \multicolumn{7}{c}{ CATEGORIA CORRECTA } \\
\cline { 2 - 9 } & Alegría & Miedo & Cólera & Tristeza & Asco & Vergüenza & Culpa & Total \\
\hline Alegría & 98.1 & 3.7 & 1.9 & 0.0 & 0.0 & 3.7 & 0.0 & 15.3 \\
Miedo & 0.0 & 68.5 & 18.5 & 9.3 & 9.4 & 0.0 & 31.5 & 19.5 \\
Cólera & 0.0 & 11.1 & 75.9 & 0.0 & 5.7 & 0.0 & 0.0 & 13.2 \\
Tristeza & 1.9 & 1.9 & 1.9 & $\mathbf{8 1 . 5}$ & 5.7 & 1.9 & 9.3 & 14.8 \\
Asco & 0.0 & 0.0 & 0.0 & 0.0 & 60.4 & 1.9 & 1.9 & 9.0 \\
Vergüenza & 0.0 & 5.6 & 0.0 & 3.7 & 3.8 & 90.7 & 16.7 & 17.1 \\
Culpa & 0.0 & 9.3 & 1.9 & 5.6 & 15.1 & 1.9 & 40.7 & 10.5 \\
No reconoce & 0.0 & 0.0 & 0.0 & 0.0 & 1.9 & 0.0 & 0.0 & 0.2 \\
\hline
\end{tabular}

Sin embargo, resulta bastante llamativa la existencia de manifestaciones que permiten un altísimo reconocimiento, superior incluso al obtenido por la combinación de cuatro síntomas, es el caso de reír para alegría, chillar para cólera y llorar para tristeza, todos ellos cambios expresivos no verbales (ver tabla IV). En el caso de miedo, en cuya codificación no aparecía ningún cambio específico (no compartido) entre los cuatro de frecuencia relativa mayor, fue mejor reconocida a partir de la manifestación tensión musucular (.76) que a partir de la combinación de cuatro cambios (.68), así este cambio que en el estudio anterior se indicó tanto para miedo (.52) como para cólera (.45), en la decodificación fue asociado a miedo en una proporción mucho mayor que a cólera (.17), resultados inversos se obtienen con aceleración del ritmo cardiaco y cambios en la respiración.

\section{TABLA IV}

Matriz completa de datos con las proporciones de elección de cada emoción ante cada manifestación presentada de forma aislada (en negrita se representan los valores de las proporciones de aquellas manifestaciones que fueron elegidas en el primer estudio para cada emoción)

\begin{tabular}{|c|c|c|c|c|c|c|c|c|c|c|c|c|c|c|c|}
\hline \multirow[b]{2}{*}{ Emoción } & \multicolumn{15}{|c|}{ CAMBIOS CORPORALES Y EXPRESIVOS } \\
\hline & 1B & $2 \mathrm{~B}$ & $3 \mathrm{~B}$ & 5B & $6 \mathrm{~B}$ & $7 \mathrm{~B}$ & $8 \mathrm{~B}$ & 10B & IE & $2 \mathrm{E}$ & $3 \mathbf{E}$ & $4 E$ & $6 \mathrm{E}$ & $1 \mathbf{V}$ & $4 \mathrm{~V}$ \\
\hline Alegria & .00 & .04 & .00 & .96 & .03 & .07 & .00 & .00 & 1.00 & .00 & .10 & .05 & .28 & .00 & .71 \\
\hline Miedo & .20 & .30 & .11 & .02 & .00 & .33 & .76 & .30 & .00 & .02 & .04 & .13 & .00 & .02 & .02 \\
\hline Cölera & .00 & .61 & .09 & .00 & .00 & .50 & .17 & .11 & .00 & .00 & .14 & .81 & .20 & .02 & -11 \\
\hline Tristeza & .44 & .00 & .06 & .02 & .01 & .00 & .00 & .00 & .00 & .98 & .10 & .00 & .02 & .18 & .02 \\
\hline Asco & .02 & .00 & .61 & .00 & .00 & .00 & .00 & .00 & .00 & .00 & .49 & .00 & .41 & .04 & .00 \\
\hline Vergüenza & .17 & .00 & .04 & .00 & .90 & .06 & .04 & .38 & .00 & .00 & .04 & .00 & .07 & .43 & .04 \\
\hline Culpa & .17 & .06 & .09 & .00 & .03 & .04 & .04 & .21 & .00 & .00 & .08 & .00 & .02 & .31 & .10 \\
\hline
\end{tabular}


Otra importante diferencia entre la decodificación de cambios y la decodificación del estudio anterior, es la que se produce en tristeza. Así mientras en el anterior estudio el cambio más informado fue el silencio (.82), seguido de llorar (.47), en el segundo estudio, las proporciones se invierten, pareciendo en primer lugar llorar (.98), después nudo en la garganta (.44) y en tercer lugar, silencio (.18). Esto parece indicar que existen algunas diferencias entre la codificación verbal de experiencias emocionales pasadas y la decodificación de cambios expresivos y corporales.

\section{DISCUSION}

A partir de estos resultados exploratorios se nos plantean diversos interrogantes. ¿ Porqué existen esas diferencias entre los cambios que indican los sujetos que experimentaron en una determinada experiencia emocional y el grado de reconocimiento que esos mismos cambios produce en decodificadores? ¿Qué significa que un sujeto nos indique un determinado cambio asociado con una experiencia emocional pasada? ¿ Realmente sintió ese cambio? ¿O simplemente sabe que está asociado a ese tipo de situación? ¿Y si es así porqué no ocurre lo mismo en el proceso de decodificación? ¿Cómo influye el recuerdo de la situación y de la experiencia emocional en autoinformes posteriores? ¿Es realmente posible recordar los cambios asociados a una experiencia emocional concreta vivida tiempo atrás? ¿Qué significa que un conjunto de sujetos asignen una emoción a un cambio corporal o expresivo determinado o a un conjunto de ellos? ¿Significa, tal vez, que realizan el proceso de reconocimiento emocional en base a sus propias experiencias emocionales? ¿O quizá que lo realizan en función de las manifestaciones externas observadas en los demás? ¿ $\mathrm{O}$ incluso que se realizan en función de representaciones sociales de las emociones y por ello, a veces, no coinciden los resultados de reconocimiento, probablemente más influidos socialmente, con los resultados de los informes que realizan los sujetos?

Como se ve no solamente se plantean cuestiones metodológicas, relacionadas con la utilización de técnicas de autoinforme, sino que también estos resultados replantean algunas de las cuestiones teóricas en el estudio de la experiencia emocional. En cualquier caso, consideramos útil, la técnica utilizada, en tanto en cuanto, aporta información de cómo los sujetos perciben $y / o$ recuerdan las manifestaciones asociadas a sus experiencias emocionales y de cómo reconocemos las emociones en los otros a partir de las descripciones verbales de estas manifestaciones.

\section{Referencias}

AEBISCHER, V., y WALLBOTT, H. G. (1986). «Measuring emotional experiences: questionnaire design and procedure, and the nature of the samplen. En K. R. Scherer, H. G. Wallbott $y$ A. B. Summerfield (Eds.), Experiencing emotion: $A$ cross-cultural study, Cambridge: Cambridge University Press.

JAMES, W. (1884). "What is an emotion? Mind, 9:188-205.

CANNON, W. B. (1915). Bodily changes in panic, bunger, fear and rage. Nueva York: Appleton-Century-Crofts. 
COSNIER, J.; FERNÁNDEZ DOLS, J. M., y JIMÉNEZ, A. (1986). «The verbalisation of emotional experiences». En K. R. Scherer, H. G. Hallbott y A. B. Summerfield (Eds.), Experiencing emotion: A cross-cultural study. Cambridge: Cambridge University Press.

DARWIN, Ch. (1872). The expression of emotions in man and animals. Londres: John Murray.

ELLGRING, H., y BNNINGER-HUBER, E. (1986). «The coding of reported emotional experiences: antecedents and reactions». En K. R. Scherer, H. G. Wallbott y A. B. Summerfield (Eds.), Experiencing emotion: A cross-cultural study, Cambridge: Cambridge University Press.

Ekman, P. (1972). Emotion in the buman face. Nueva York: Pergamon.

FERNÁNDEZ DOLS, J. M., y ORTEGA, J. E. (1985). «Los niveles de análisis de la emoción: James cien años despuésm. Estudios de Psicología, 21:35-56.

Iglesias, J.; NARANJO, J. M.; PICAZO, J. M., y ORTEGA, J. E. (1984). «La cara y la emoción: Datos para una réplican. Estudios de Psicología, 18:101-111.

IZARD, C. E. (1971). The face of emotion. Nueva York: Appleton-Century-Crofts.

JIMÉNEZ, A. (en prensa). Marcadores emocionales en la conducta vocal. Madrid: Universidad Autónoma de Madrid.

KEMPER, T. D. (1978). "Power, status and emotions: A sociological contribution to a psychophysiological domain». En K. R. Scherer y P. Ekman (Eds.), Approaches to emotion. Hillsdale N. J.: Erlbaum.

LAZARUS, R. S. (1982). *Thoughts on the relations between emotion and cognition". American Psychologist, 37:1019-1024.

PlutchiK, R.; y Kellerman, H. (Eds.) (1980). Emotion, theory, research and experience I: Theories of emotion. Nueva York: Academic.

RIMÉ, B., y GIOVANNINI, D. (1986). *The physiological patterns of reported emotional statesn. En K. R. Scherer, H. G. Wallbott y A. B. Summerfield (Eds.), Experiencing emotion: $A$ cross-cultural study. Cambridge: Cambridge University Press.

SCHERER, K. R. (1982). "Emotion as a process: Function, origin and regulation». Social Science Information, 21:555-570.

SCHERER; K. R. (1984). *On the nature and function of emotion: A component process approach». En K. R. Scherer y P. Ekman (Eds.), Approaches to emotion. Hillsdale, N. J.: Erlbaum.

SCHERER, K. R. (1986). «Studying emotion empirically: Issues and a paradigma for research». En K. R. Scherer, H. G. Wallbott y A. B. Summerfield (Eds.), Experiencing emotion: $A$ cross-cultural study. Cambridge: Cambridge University Press.

SCHERER, K. R.; WALlBOTT, H. C., y SUMmerfield, A. B. (Eds.). (1986), Experiencing emotion: $A$ cross-cultural study. Cambridge: Cambridge University Press.

TomKINS, S. S. (1962-1963). Affect, imagery, consciousness (2 vol). Nueva York: Springer.

TURREGROSA, J. R. (1984). «Emociones, sentimientos y estructura social». En J. R. Torregrosa y E Crespo (Eds.), Estudios básicos de Psicología Social. Barcelona: Hora.

WAllBotT, H. G.; RICCI-BITTI, P., y BNNINGER-HUBER, E. (1986). "Non-verbal reactions of emotionai ?xperiences". En K. R. Scherer, H. G. Wallbott y A. B. Summerfield (Eds.), Experiencing emolion: A cross-cultural study. Cambridge: Cambridge University Press.

VALLE INCLÁN, F. (198j; , «a cara y la emoción: Límites de la universalidad». Estudios de Psicologia, 16:107-115.

VALLE INCLÁN, F. (1985). «Universalidad de la expresión emocional e inferencia correcta: Una contestación a Iglesias y cols. (1984)m. Estudios de Psicología, 21:91-97.

WUNDT, W. (1905). Grundzüge der physiologischen Psychologie, (vol. 3). Leipzig: Wilhelm Engelmann.

ZAJONC, R. B. (1980). «Feeling and thinking: Preferences need no inferences": Américan Psychologist, 35:151-175. 